\title{
On the Cohomology of the 2-connected Cover of the Loop Space of Simple Lie Groups
}

Dedicated to Professor Nagayoshi Iwahori on his 60 th birthday

By

\section{Akira KONO*}

\section{§1. Introduction}

Let $G$ be a compact, connected, simply connected, simple, Lie group, $p$ a prime and $t \in H^{2}\left(\Omega G ; \mathbb{F}_{p}\right)$ a generator. Let $x_{3} \in H^{3}\left(G ; \mathbb{F}_{p}\right)$ be a generator satisfying $\sigma\left(x_{3}\right)=t$, where $\sigma$ is the cohomology suspension. Since $G$ is compact and $H^{*}\left(\Omega G ; \mathbb{F}_{p}\right)$ is a Hopf algebra, there exists an integer $d=d(G, p)$ satisfying

$$
t^{p^{d}-1} \neq 0 \text { and } t^{p^{d}}=0 \text {. }
$$

Let $G\langle 3\rangle$ be the 3 -connected cover of $G$. For a graded module $A=\oplus A_{i}$ of finite type over $\mathbb{F}_{p}$, we define $P(A, q)=\sum\left(\operatorname{dim} A_{i}\right) q^{i}$. The purpose of this note is to determine $P\left(H^{*}\left(\Omega G\langle 3\rangle ; \mathbb{F}_{p}\right), q\right)$. There is a fibering (*) $S^{1} \rightarrow \Omega G\langle 3\rangle \rightarrow \Omega G$. Since $H^{*}\left(\Omega G ; \mathbb{F}_{p}\right)$ is a Hopf algebra, there exists a graded algebra $A(G, p)$ satisfying

$$
H^{*}\left(\Omega G ; \mathbb{F}_{p}\right)=\mathbb{F}_{p}[t] /\left(t^{p^{d}}\right) \otimes A(G, p) \text {. }
$$

On the other hand $H^{*}(\Omega G ; \mathbb{Z}$ ) is torsion free (cf。Bott [8]) and so

$$
P(A(G, p), q)^{-1}=\left(\prod_{j=2}^{l}\left(1-q^{2 m(j)}\right)\right) \cdot\left(1-q^{2 a(G, p)}\right)
$$

where $a(G, p)=p^{d}$ and $m(1)=1<m(2) \leq \cdots \leq m(l)$ are the exponent of the weyl group of $G$. Using the Gysin exact sequence for (*) we have

Theorem 1. $P\left(H^{*}\left(\Omega G\langle 3\rangle ;{ }_{p}\right), q\right)=P(A(G, q), q) \circ\left(1+q^{2 a(G, p)-1}\right)$ 。

On the other hand the number $d(G, p)$ is given by

Communicated by N. Shimada, October 28, 1985.

* Department of Mathematics, Kyoto University, Kyoto 606, Japan. 
Theorem 2. (1) For a classical type $G$,

$$
d(G, p)=\left\{\begin{array}{l}
r(n, p) \quad \text { if } G=S U(n), \\
r([n / 2], 2) \quad \text { if } G=\operatorname{Spin}(n) \text { and } p=2, \\
r(2 n, p) \quad \text { if } G=\operatorname{Spin}(2 n+1), \operatorname{Spin}(2 n) \text { or } \\
\quad S p(n) \text { and } p \text { is an odd prime, }
\end{array}\right.
$$

where $p^{r(n, p)-1}<n \leq p^{r(n, p)}$ and $d(G, p)=1$ if $G=S p(n)$ and $p=2$.

(2) $d(G, 2)=2$ if $G=G_{2}$ or $\mathrm{F}_{4}$ and $d(G, 2)=4$ if $G=E_{6}, E_{7}$ or $E_{8}$.

(3) For an odd prime $p, d(G, p)$ is given by the following table:

\begin{tabular}{|c|c|c|c|c|c|c|c|c|c|c|}
\hline$G$ & \multicolumn{2}{|c|}{$G_{2}$} & $F_{4}, E_{6}$ & \multicolumn{3}{|c|}{$E_{7}$} \\
\hline$p$ & 5 & $\neq 5$ & $\leq 11$ & $>11$ & 3 & $5 \leq p \leq 17$ & $>17$ & 3 & $5 \leq p \leq 29$ & $>29$ \\
\hline$d(G, p)$ & 2 & 1 & 2 & 1 & 3 & 2 & 1 & 3 & 2 & 1 \\
\hline
\end{tabular}

\section{\$2. Proof of Theorem 2}

In this section $P(k)=\mathscr{P}^{p^{k-1}} \ldots \mathscr{P}^{1}\left(\mathscr{P}^{i}=S q^{2 i}\right.$ if $\left.p=2\right)$. Put $E=\left\{G_{2}\right.$, $\left.F_{4}, E_{6}, E_{7}, E_{8}\right\}$ and $P T=\{(G, 2) ; G \in E\} \cup\left\{(G, 3) ; G \in E, G \neq G_{2}\right\} \cup\left\{\left(E_{8}\right.\right.$, 5) $\} \cup\{(\operatorname{Spin}(n), 2) ; n \geq 7\}$. For a compact 1-connected simple Lie group $G, H^{*}(G ; Z)$ is $p$-torsion free if and only if $(G, p) \notin P T$.

Lemma 1. If $P(k) x_{3}$ is decomposable, then $t^{p^{k}}=0$.

Proof. $t^{p^{k}}=P(k) t=P(k) \sigma\left(x_{3}\right)=\sigma\left(P(k) x_{3}\right)=0$.

Lemma 2. For any $G, P(d(G, p)) x_{3}$ is decomposable.

Proof. Since $\operatorname{deg} P(d) x_{3}=2 a+1$, we need only show $H^{2 a+1}\left(G ; \boldsymbol{F}_{p}\right)$ is decomposable, which is shown in [4] and [7] for $G \notin E$ and in [1], [2], [3] and [6] for $G \in E$.

Lemma 3. If $H^{*}(G ; \mathbb{Z})$ is $p$-torsion free and $P(k) x_{3} \neq 0$, then $t^{p^{k}} \neq 0$.

Proof. Since $H^{*}(G ; \mathbb{Z})$ is $p$-torsion free, as an algebra

$$
H_{*}\left(G ; F_{p}\right)=\Lambda\left(y_{3}, y_{2 m(2)+1}, \ldots, y_{2 m(l)+1}\right)
$$

where $\operatorname{deg} y_{j}=j$ and $y_{3}$ is the dual of $x_{3}$. Since $P(k) x_{3} \neq 0$ and $P(k) x_{3}$ 
is primitive, the dual of $P(k) x_{3}$ is not decomposable and so there exists $y_{u}\left(u=2 p^{k}+1\right)$ such that $P(k)_{*} y_{u}=y_{3}$ (cf. [17]). On the other hand

$$
H_{*}\left(\Omega G ; \mathbb{F}_{p}\right)=\mathbb{F}_{p}\left[s_{2}, s_{2 m(2)}, \ldots, s_{2 m(l)}\right]
$$

where $\operatorname{deg} s_{j}=j . \quad s_{j}=\tau^{\prime}\left(y_{j+1}\right) \quad\left(\tau^{\prime}\right.$ is the homology transgression) and $s_{2}$ is the dual of $t$. By the naturalities of $\tau^{\prime}$ we have $P(k)_{*} s_{u-1}=s_{2}$ mod decomposables and so $P(k)_{*} s_{u-1}=s_{2}$ and so $t^{p^{k}}=P(k) t \neq 0$.

Lemma 4. If $(G, p)=(\operatorname{Spin}(n), 2)$, then $t^{2^{d(G, p)-1}} \neq 0$ 。

Proof. Since $H^{*}\left(G ; \mathbb{F}_{2}\right)$ is a polynomial algebra for $* \leq n-1$ (cf. [4] or Theorem 3.2 of [10]), the cohomology suspension $\sigma$ induces a monomorphism $\bar{\sigma}: Q H^{*}\left(G ; \mathbb{F}_{2}\right) \rightarrow H^{*-1}\left(\Omega G ; \mathbb{F}_{2}\right)$ for $* \leq n-1$, where $Q$ denotes the indecomposable quotient. Since $P(d(G, 2)-1) x_{3}$ is not decomposables by [3], we have

$$
t^{2^{d-1}}=P(d-1) t=P(d-1) \bar{\sigma}\left(x_{3}\right)=\bar{\sigma}\left(P(d-1) x_{3}\right) \neq 0 。
$$

Lemma 5. For any $(G, p) \notin P T, P(d-1) x_{3}$ is not decomposable.

Proof. See [6] for $G \notin E$ and [18] for $G \in E$.

Lemma 6. For any $(G, p) \in P T,(G, p) \neq(\operatorname{Spin}(n), 2), t^{p^{d-1}} \neq 0$ 。

Proof. If $p$ is an odd prime we can easily show $H^{2 k-1}\left(\Omega G\langle 3\rangle ; \mathbb{F}_{p}\right)$ $=0$ for $k \leq p^{d-1}$ by making use of the structure of $H^{*}\left(G\langle 3\rangle ; \mathbb{F}_{p}\right.$ ) (cf。 $[11],[13],[17])$. If $p=2, H_{*}\left(\Omega G\langle 3\rangle ; \mathbb{F}_{2}\right)$ is known (see [15]). (See also [14]).

Proof of Theorem 2. By Lemma 1 and Lemma 2, $t^{p^{d}}=0$. Therefore we need only show $t^{p^{d-1}} \neq 0$, since $H^{*}\left(\Omega G ; F_{p}\right)$ is a Hopf algebra. It is shown by Lemma 3, Lemma 4, Lemma 5 and Lemma 6.

Remark 7. (1) Since $P(k) x_{3}$ is primitive and $\operatorname{deg} P(k) x_{3}$ is odd, $P(k) x_{3}$ is decomposable if and only if $P(k) x_{3}=0$.

(2) If $H^{*}(G ; \mathbb{Z})$ is $p$-torsion free, then $t^{p^{k}} \neq 0$ if and only if $P(k) x_{3} \neq 0$ by Lemma 1 and Lemma 3. Moreover by Theorem 2, $t^{p^{k}} \neq 0$ if and 
only if $P(k) x_{3} \neq 0$ for any $G$.

Remark 8. If $G=S U(n)$ or $S p i n(n)$, we can prove $t^{p^{d-1}} \neq 0$ by making use of the following (cf. Bott [9] 8-10): There exist maps $j: X_{n}=\boldsymbol{C P}^{n-1} \rightarrow \Omega S U(n)$ and $j^{\prime}: X_{n}^{\prime}=S O(n+1) / S O(n-1) \times S O(2) \rightarrow \Omega S p i n(n)$

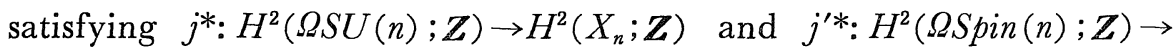
$H^{2}\left(X_{n}^{\prime} ; \mathbb{Z}\right)$ are isomorphic.

\section{Acknowledgement}

Theorem 1 is a special case of a general theory of V.G. Kac and D.H. Peterson on the topology of Kac-Moody Lie groups. They also computed $d(G, p)$ in terms of the Affine Weyl group of $G$ and showed that $d(G, p)=1$ if $p>m(l)$. Their work has prompted me to write this paper。 (See [19].)

\section{References}

[1] S. Araki, Differential Hopf algebra and the cohomology mod 3 of the compact exceptional groups $E_{7}$ and $E_{8}$, Ann. Math., 73 (1961), 404-436.

[2] Cohomology modulo 2 of the compact exceptional groups $E_{6}$ and $E_{7}, J$. Math. Osaka City Univ., 12 (1961), 43-65.

[3] S. Araki and Y. Shikata, Cohomology mod 2 of the compact exceptional group $E_{8}$, Proc. Japan Acad., 37 (1961), 619-622.

[4] A. Borel, Sur l'homologie et la cohomologie des groupes de Lie compacts connexes, Amer. J. Math., 76 (1954), 273-342.

[5] Topology of Lie groups and characteristic classes, Bull. A.M.S., 61 (1955), $394-432$.

[6] Sous groupes commutatifs et torsion des groupes de Lie compacts connexes, Tohoku Math. J., 13 (1961), 216-240.

[7] A. Borel and J.P. Serre, Groupes de Lie et puissances reduites de Steenrod, Amer.J. Math., 73 (1953), 409-448.

[8] R. Bott, An application of the Morse theory to the topology of Lie groups, Bull. Soc. Math. France, 84 (1956), 251-281.

[9] - The space of loops on a Lie group, Michigan Math. J., 5 (1958), 35-61.

[10] K. Ishitoya, A. Kono and H. Toda, Hopf algebra structure of mod 2 cohomology of simple Lie groups, Publ. R. I.M.S. Kyoto Univ., 12 (1976), 141-167.

[11] A. Kono, Hopf algebra structure of simple Lie groups, J. Math. Kyoto Univ., 17 (1977), 259-298.

[12] - Hopf algebra structure and cohomology operations of the mod 2 cohomology of the exceptional Lie groups, Japan. J. Math., 3 (1977), 49-55.

[13] A. Kono and M. Mimura, Cohomology operations and the Hopf algebra structure of the compact, exceptional Lie groups $E_{7}$ and $E_{8}$, Proc. London Math. Soc., 35 (1977), 345-358. 
[14] A. Kono, On the cohomology of exceptional Lie groups, Algebraic and Topological Theories, Kinokuniya, Japan, 1985, 328-338.

[15] K. Kozima, Mod 2 homology ring of the space of loops on exceptional Lie groups, (in Japanese).

[16] J. Milnor and J. Moore, On the structure of Hopf algebra, Ann. Math., 81 (1965), 211-264.

[17] M. Mimura, Homotopy groups of Lie groups of low rank, J. Math. Kyoto Univ., 6 (1967), 131-176.

[18] M. Mimura and H. Toda, Cohomology operations and the homotopy of compact Lie groups I, Topology, 9 (1970), 317-336.

[19] V. G. Kac, Torsion in cohomology of compact Lie groups and Chow rings of reductive algebraic groups, Inv. Math., 80 (1985), 69-79. 
